\title{
A SUPERMASSIVE OBJECT IN THE NUCLEUS OF NGC 4258?
}

L. J. GREENHILL, D. R. JIANG, J. M. MORAN AND M. J. REID

Harvard-Smithsonian Center for Astrophysics

60 Garden St, Cambridge, MA 02198 USA

K.-Y. LO

Department of Astronomy, University of Illinois

1002 W. Green St, Urbana, IL 61801 USA

M. J. CLAUSSEN

National Radio Astronomy Observatory

P. O. Box O, Socorro, NM 87801 USA

C. HENKEL AND T. L. WILSON

Max-Planck-Institut für Radioastronomie

Auf dem Hugel 69, D-53121 Bonn, Germany

R. BECKER

GKSS Forschungszentrum, Institut für Physik

Max-Planck-Strasse, D-21502 Geesthacht, Germany

AND

J. G. A. WOUTERLOOT

I. Physikalisches Institut

Universität zu Köln, Zülpicher Str. 77, D-50997 Köln, Germany

Abstract.

VLBI images of the $\mathrm{H}_{2} \mathrm{O}$ megamaser in NGC 4258 and a time series of spectra taken over several years combine to make a compelling case that there is a compact molecular disk associated with a supermassive object in the nucleus of this galaxy. The images of the maser in the velocity range near the systemic velocity show a highly elongated structure with a major axis of about $0.009 \mathrm{pc}$, along which the gradient in line-of-sight velocity is essentially a constant of $7970 \pm 40 \mathrm{~km} \mathrm{~s}^{-1} \mathrm{pc}^{-1}$. The observed acceleration of these spectral features by about 6-11 $\mathrm{km} \mathrm{s}^{-1} \mathrm{yr}^{-1}$, the presence of highvelocity maser satellite emission, and the VLBI results suggest emission 
from a disk of diameter $0.2 \mathrm{pc}$, rotating with a velocity of $\sim 800 \mathrm{~km} \mathrm{~s}^{-1}$, bound by a mass of $1.5 \times 10^{7} \mathrm{M}_{\odot}$ and density of $\gtrsim 3.6 \times 10^{9} \mathrm{M}_{\odot} \mathrm{pc}^{-3}$.

\section{Introduction}

The $\mathrm{H}_{2} \mathrm{O}$ maser in NGC 4258, with an apparent luminosity $>50 L_{\odot}$ on the assumption of isotropic emission of radiation, is among 13 known extragalactic $\mathrm{H}_{2} \mathrm{O}$ "megamasers". Subarcsecond astrometry shows that at least three are associated with galactic nuclei and that the maser emission arises at projected distances of less than a few parsecs from the compact radio continuum sources (Claussen \& Lo 1986). There is no accepted mechanism that explains the extraordinary apparent luminosity of these megamaser sources. They either mark individual sites of star formation, as in our galaxy, or sites of energetic interactions between outflows (e.g., jets) or intense high-energy radiation fields (e.g. X-ray) and ambient material (Elitzur 1992, Neufeld et al. 1994).

The megamaser in NGC 4258 is a potentially important diagnostic tool for understanding the galaxy's active nucleus, which contains a radio jet and a twisted optical jet (Cecil et al. 1992) whose orientation is almost north-south within the innermost $1 \mathrm{kpc}$ of the nucleus (Cecil et al. 1995). We assume a distance of $7 \mathrm{Mpc}$ for the galaxy (cf. Courtès et al. 1993).

Recently, Nakai et al. (1993) discovered high-velocity maser emission symmetrically offset from the systemic velocity by about $\pm 800 \mathrm{~km} \mathrm{~s}^{-1}$, with the redshifted emission arising within $0.05(1.7 \mathrm{pc})$ of the emission at the systemic velocity of the galaxy. In addition, many of the low-velocity maser features appear to drift in concert by about $6-11 \mathrm{~km} \mathrm{~s}^{-1} \mathrm{yr}^{-1}$ (Haschick et al. 1994, Greenhill et al. 1995a). Features fade away at the high velocity end of the systemic velocity group and new ones appear at the low velocity end such that the mean velocity of the group remains nearly constant and close to the systemic velocity of the galaxy.

\section{Evidence for a Disk}

We observed the megamaser with a 4 station VLB array. Preliminary reduction of the data was presented by Claussen et al. (1988). Synthesis imaging shows that the spectral features are spatially unresolved and distributed along a line in the sky, subtending about $260 \mu$ as in angle $(0.009 \mathrm{pc})$ at a position angle of $83^{\circ} \pm 2^{\circ}$ (Figure 1). The distribution of the maser emission exhibits a linear gradient of position with line-of-sight velocity of 3.70 $\pm 0.02 \mu \mathrm{as} \mathrm{km} \mathrm{km}^{-1} \mathrm{~s}$ or $7970 \pm 40 \mathrm{~km} \mathrm{~s}^{-1} \mathrm{pc}^{-1}$ along the major axis (Figure 2). The elongation and velocity gradient suggests the maser emission lies in 
a rotating disk that is viewed edge-on. In the model, maser emission traces only a small arc along the near side of the disk, where it amplifies continuum emission from the massive central source. The high-velocity emission arises at the edges of the disk where the line of sight is tangent to it (Watson \& Wallin 1994, Greenhill et al. 1995b).

The radius of the disk, extrapolated from the gradient, is $\sim 0.1 \mathrm{pc}$, where the rotational velocity of the disk at this radius is about $800 \mathrm{~km} \mathrm{~s}^{-1}$. The anticipated drift in the velocities of the low-velocity spectral features $\left(V^{2} / R\right)$ is about $7 \mathrm{~km} \mathrm{~s}^{-1} \mathrm{yr}^{-1}$. This is close to the observed values of $6-11 \mathrm{~km} \mathrm{~s}^{-1} \mathrm{yr}^{-1}$ (Haschick et al. 1994 and Greenhill et al. 1995a), and is inversely proportional to the assumed distance to the galaxy. Of equal importance, the drift of the high-velocity features is $\lesssim 1 \mathrm{~km} \mathrm{~s}^{-1} \mathrm{yr}^{-1}$, as expected for the disk model (Greenhill et al. 1995a). It is unlikely that the acceleration of spectral features is an artifact of relative intensity variations among blended lines. The binding mass for this system is $1.4 \times 10^{7} \mathrm{M}_{\odot}$, and the mass of the gas in the disk is $\lesssim 10^{6} \mathrm{M}_{\odot}$, precludes solid-body rotation. The tight correlation of position and velocity suggests that the maser emission is confined to a thin annulus, of faction radial thickness $\lesssim 0.2$. This width together with a $50 \mu$ as upper limit on the north-south extent of the emission limits the inclination of the disk to $\lesssim 5^{\circ}$. A revised distance of $5.4 \pm 1.0 \mathrm{Mpc}$ can be computed based on the geometry of this disk model.

The diameter of the molecular ring is smaller than that often predicted for active nuclei (Antonucci 1993, and references therein). However, Gwinn et al. (1993) may also have evidence, from $\mathrm{H}_{2} \mathrm{O}$ maser emission, for a disk of similar dimension in the Seyfert NGC 1068. The strongest confirmation of the disk model for NGC 4258 will be the VLBI measurements of the relative positions and proper motions of the low and high-velocity emission.

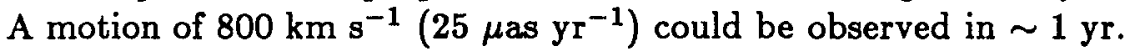

\section{References}

Antonucci, R. J. 1993, Ann. Rev. A\&A, 31, 473

Cecil, G., Wilson, A. S., \& De Pree, C. 1995, ApJ, 440, in press

Cecil, G., Wilson, A. S., \& Tully, R. B. 1992, ApJ 390, 365

Claussen, M.J. \& Lo, K.-Y., 1986, ApJ 308, 592

Claussen, M.J., Reid, M.J., Schneps, M.H., Moran, J.M., \& Güsten, R., 1988, in "The Impact of VLBI on Astrophysics and Geophysics," IAU Symp. 129, 231

Courtès, G., Petit, H., Hua, C.T., Martin, P., Blecha, A., Huguenin, D., \& Golay, M. 1993, A\&A, 268, 419

Elitzur, M., 1992, Astronomical Masers, (Boston: Kluwer), chap. 13

Greenhill, L. J., Henkel, C., Becker, R., Wilson, T. L., Wouterloot, J. G. A. 1995a, A\&A, in prep.

Greenhill, L. J., Jiang, D. R., Moran, J. M., Claussen, M. J., \& K.-Y. Lo 1995b, ApJ, 440, in press

Gwinn, C. R., Antonucci, R. J., Barvainis, R., Ulvestad, J., \& Neff, S. 1993, in "Subarc- 
second Radio Astronomy," ed. R. J. Davies \& R. S. Booth (Cambridge: Cambridge Univ. P), 331

Haschick, A. D., Baan, W. A., Peng, E. W. 1994, ApJ, in press

Nakai, N., Inoue, M., \& Miyoshi, M., 1993, Nat 361, 45

Neufeld, D. A., Maloney, P. R., \& Conger, S. 1994, ApJL, in press

Watson, W. D. \& Wallin, B. K. 1994, ApJ, 432, L35

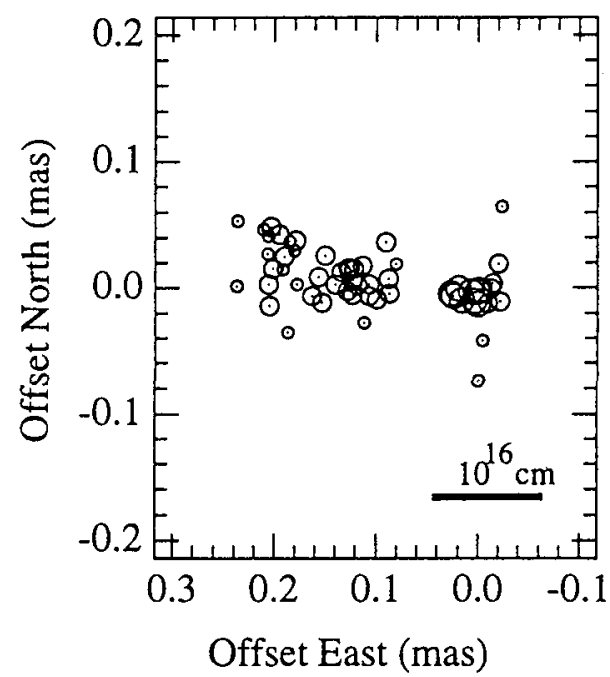

Figure 1. - Distribution of megamaser emission from VLBI observations with resolution $\sim 0.1$ mas. Each circle represents emission over $1.3 \mathrm{~km} / \mathrm{s}$. The areas are proportional to the peak flux densities.

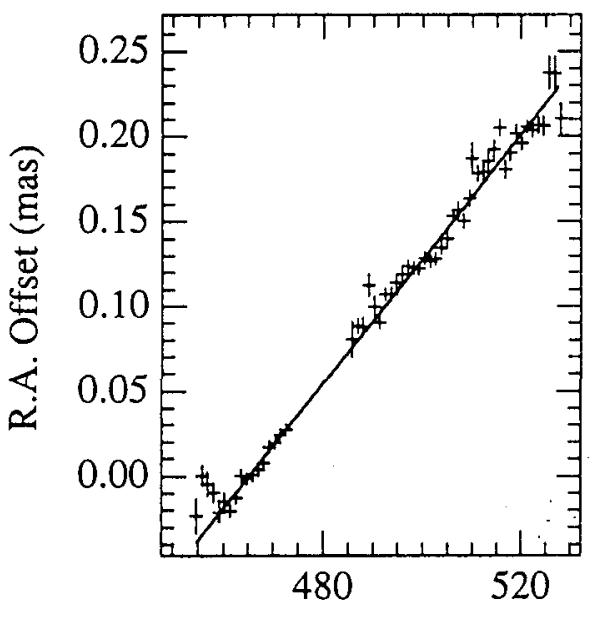

LSR Velocity $\left(\mathrm{km} \mathrm{s}^{-1}\right)$

Figure 2. - Position-velocity gradient in the megamaser emission. The fitted line has a slope of $3.67 \mu \mathrm{as} / \mathrm{km} / \mathrm{s}$.

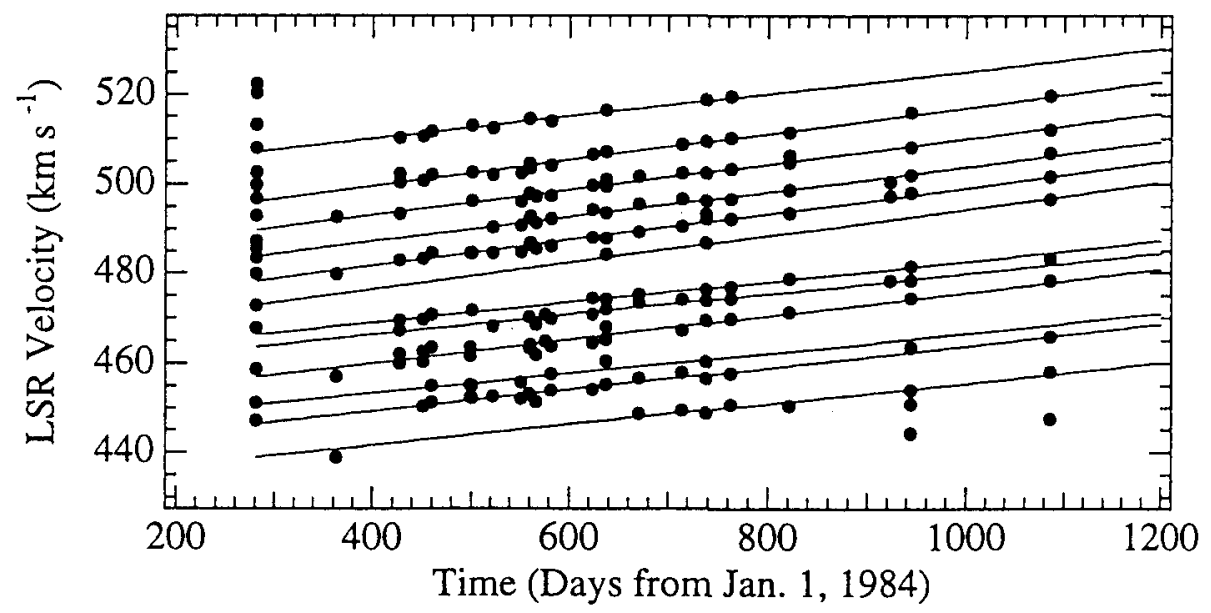

Figure 3. Velocities of 12 maser features followed through 20 epochs over 2 years. The average drift is $9.5 \mathrm{~km} / \mathrm{s} / \mathrm{yr}$. 